This study was designed to describe the clinicopathological characteristics of cervical squamous cell carcinoma (SCC) intra epithelial spreading to the upper genital tract.

A total of fifteen patients were enrolled, including eleven patients which were cervical SCC spreading to the endometrium, four patients whose squamous cell carcinoma intra epithelial spread along the uterine cavity to the adnexa. Clinical information and pathological data were collected. Human high risk HPV was detected by mRNA in situ hybridization.

The average age was 55.07 years old. Twelve of the fifteen patients were post menopause women. The intra epithelial spread pattern can be seen in thirteen patients. Five of the fourteen patients received a uterine apoxesis, the other pre operative condition including chemotherapy, papillary thyroid carcinoma, thyroid hypofunction, and immune deficiency. HPV was positive in all the patients' specimen, and the infection status of cervical lesions, endometrial lesions, and adnexal lesions were same. For the peculiar case twelve, the cervical CIS lesion intra epithelial spread along the mucosa of uterine cavity and fallopian tube, and invasive SCC can be identified in the uterine corpus, fallopian tubes and ovaries.

Intra epithelial spread pattern is an indolent spreading pathway of the cervical SCC, it can involve the upper uterine tract even when the cervical lesion in at a very early stage, such as CIS. The deepest invasion site may not be the primary site. The intra epithelial spread may have relationship with pre operative disposal. High risk HPV detection can help to confirm the cervical origination.
IGCS20_1279

\section{HISTOPATHOLOGIC ULTRASTAGING OF SENTINEL LYMPH NODES IN ENDOMETRIAL CARCINOMA: A RETROSPECTIVE ANALYSIS OF THE TUEBINGEN WOMEN'S HOSPITAL 2006-2016 PATIENT COHORT}

${ }^{1} \mathrm{~L}$ Baudoux*, ${ }^{2} \mathrm{~A}$ Staebler, ${ }^{2} \mathrm{~K}$ Greif, ${ }^{1} \mathrm{~B}$ Krämer, ${ }^{1} \mathrm{~S}$ Hoffmann, ${ }^{1} \mathrm{~F}$ Neis, ${ }^{1} \mathrm{~J}$ Andress, ${ }^{1} \mathrm{M}$ Grube, ${ }^{1} \mathrm{~J}$ Pasternak, ${ }^{3} \mathrm{~F}$ Taran, ${ }^{1} \mathrm{P}$ Krämer, ${ }^{1} \mathrm{C}$ Bachmann, ${ }^{1} \mathrm{E}$ Oberlechner, ${ }^{1} \mathrm{SY}$ Brucker, 'S Kommoss. 'Department of Women's Health, Tuebingen University Hospital, Germany; ${ }^{2}$ Institute of Pathology, Tuebingen University Hospital, Germany; ${ }^{3}$ Department of Gynecology, Zurich University Hospital, Switzerland

\subsection{6/ijgc-2020-IGCS.229}

Introduction Lymph node metastases are considered a major risk factor in endometrial carcinoma patient management and nodal assessment is an integral part of staging. While still under investigation sentinel node biopsy has been established in many centers. It has been shown that histopathologic ultrastaging leads to an increased detection of isolated tumor cells (ITC) and micrometastases (MIC). However, the clinical impact of ITC and MIC is not yet clearly defined. The aim of this study was to retrospectively perform histopathologic ultrastaging in a large series of endometrial carcinoma patients all of which have been treated at the Tuebingen University Women's Hospital.

Method Patients with sentinel node biopsy during endometrial carcinoma surgery were identified. All cases with negative sentinel nodes, were submitted to retrospective histopathologic ultrastaging $(5 \times 100 \mu \mathrm{m}$ intervals followed by $500 \mu \mathrm{m}$ sections
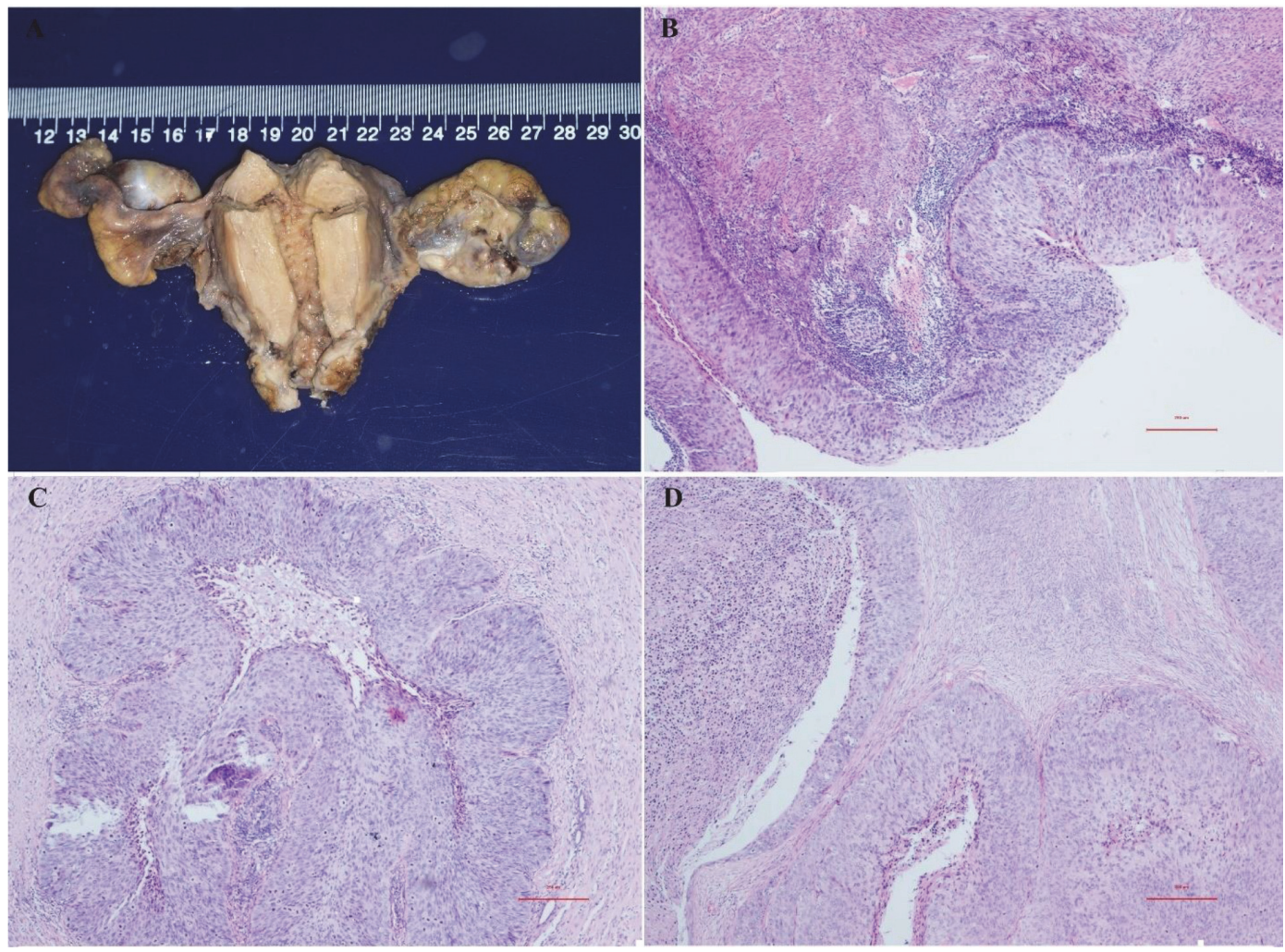

Abstract 266 Figure 1 
and immunohistochemical pancytokeratine staining of the entire lymphnode).

Results A total of $\mathrm{n}=159$ cases was available, of which in 5/ $159(3.0 \%)$ case positive nodes were detected during initial routine pathology workup (pT1a,G3: $n=1$; pT1b,G1: $n=1$; pT1b,G3: $n=2 ; p T 2, G 2: n=1)$. After retrospective ultrastaging additional micrometastases were identified in $\mathrm{n}=3$ cases (pT1a,G1; pT1a,G2; pT1a,G3), isolated tumor cells in $\mathrm{n}=2$ cases (pT1a,G3; pT1b,G3), a macrometastasis was revealed in one case (pT1b,G3). Overall $11 / 159$ cases $(6,7 \%)$ were finally reported positive.

Conclusion In our cohort histopathologic ultrastaging of endometrial carcinoma sentinel nodes led to a substantially increased detection rate of nodes with isolated tumor cells, micro- and macrometastases. We suggest that histopathologic ultrastaging should be mandatory in endometrial carcinoma sentinel lymph node workup.

\section{IGCS20_1280}

\section{PAPILLARY CARCINOMA OF THE BREAST : REPORT OF 30 CASES}

${ }^{1}$ I Bouraouiं, 'M Bouhani, 'S Sakhri, ${ }^{1} \mathrm{M}$ Slimene, ${ }^{2} \mathrm{O}$ Adouni, ${ }^{1} \mathrm{M}$ Hechiche, ${ }^{1} \mathrm{R}$ Chargui, ${ }^{1} \mathrm{~K}$ Rahal. ${ }^{1}$ Department of Oncologic Surgery, Salah Azaiez Institute, Tunisia; ${ }^{2}$ Department of anatomopathology, Salah Azaiez Institute, Tunisia

\subsection{6/ijgc-2020-IGCS.230}

Introduction Breast cancer is the most common type of cancers in women. Invasive ductual carcinoma remains the most frequent histological type but invasive papillary carcinoma (IPC) is an uncommon histopathological variant. It accounts for approximately 3 to $6 \%$ of all invasive breast cancers. Morphologically, It is characterised by pseudopapillary. IPC is associated with a high incidence of axillary lymphnode metastasis and lymphovascular invasion. Local recurrence is more frequent.

Patients and Methods The data of 70 patients were reviewed retrospectively. Thirty of them were included in our study between 2004 and 2019.

Results All patients were women and the mean age was 62 years old. Right breast was the most site involved and tumor was located mostly in the outer upper quadrant. Clinical presentation was predominantely a breast lump and axillary lympnode was detected in $76 \%$ of cases of which $20 \%$ were metastatic. Tumoral stage was T2 N1M0 in $50 \%$. Ultrasound examination revealed a cystic or solidocystic appearence in most cases. The mean histological tumor size was $26 \mathrm{~mm}$. Immunohistochemical studies revealed the positivity of hormonal receptor in $73 \%$ and the negativity of HER in all cases. Age, Lymphovascular invasion and tumor necrosis were significantly associated to recurrence free and overall survival. Local recurrence was noted in 6\%. Fiveyear Recurrence free survival and overall survival were respectively $87 \%$ and $88 \%$.

Conclusion Invasive micropapillary carcinoma has a poor clinical behaviour but in our study it has a good prognosis with a high rate of survival and less rate of recurrence.
IGCS20_1281

\section{CLINICAL PROFILES AND INTRAOPERATIVE CHARACTERISTICS AFTER CYTOREDUCTIVE SURGERY AND HYPERTHERMIC INTRAPERITONEAL CHEMOTHERAPY- AN EXPERIENCE FROM A TERTIARY CARE CENTRE IN INDIA}

N Kumar*, MD Ray, S Deo. AllMS, India

\subsection{6/ijgc-2020-IGCS.231}

Introduction Peritoneal surface malignancies were considered as terminal disease before the introduction of cytoreduction surgery (CRS) and Hyperthermic Intraperitoneal Chemotherapy (HIPEC).

Methods Ninety-seven patients underwent CRS and HIPEC from 2017 - 2019 and data was recorded from a prospective maintained computerized database. Clinical profile, Peritoneal Cancer Index (PCI), Completeness of Cytoreduction (CC), CRS Operative time and HIPEC time were reviewed.

Results Demographic characteristics-

The median age was 57 years (range; 25-65 years). Females comprised $70 \%$ of the population. The median body mass index was 27.21 (Range; 25-39).

Disease distribution-

Ovarian cancer comprised of $58.76 \%$, Colorectal constituted $32 \%$ and appendiceal cancer accounted for 9.2\%. Highgrade histology was found in 76 patients.

PCI \& CC Score characteristics-

Median Peritoneal Cancer Index (PCI) was 14 (range; 825). Completeness of Cytoreduction (CC) of 0 and 1 was achieved in all patients, with CC -0 in 87 patients (90\%) and CC-1 in 10 patients (10\%). The mean operating time for CRS was 581 minutes (range; 172-874) and for HIPEC was 59.58 minutes (range; $45-60$ ).

The extent of resection-

Right hemicolectomy along with anterior peritonectomy was done in 14 patients. Low Anterior Resection with pelvic peritonectomy was done in 10 patients. Total omentectomy, cholecystectomy and segmental ureteric resection were performed in 57, 10 and 3 patients respectively.

Conclusions Complete CRS and HIPEC are safely done with expert hands in selected patients. The intraoperative extent of disease and complete CRS along with the duration of surgery and chemotherapeutic drug dosage are important determinants of peri-perioperative outcomes.

\section{IGCS20_1282}

\section{CERVICAL CANCER AND HUMAN PAPILLOMAVIRUS AWARENESS, VACCINE STATUS AND RISK FACTORS AMONG WOMEN IN SAUDI ARABIA}

${ }^{1} \mathrm{~K}$ Akkour*, ${ }^{2} \mathrm{M}$ ARAFAH, 'L Alghosun, ${ }^{1} \mathrm{H}$ Alhalal, ${ }^{1} \mathrm{~A}$ Bassi, ${ }^{1} \mathrm{~N}$ Alayed, ${ }^{3} \mathrm{~S}$ Iqbal. ${ }^{1}$ Department of Obstetrics and Gynecology, College of Medicine, King Saud University, Saudi Arabia; ${ }^{2}$ Department of Obstetrics and Gynecology, College of Pathology, King Saud University, Saudi Arabia; ${ }^{3}$ Faculty of Medicine, Alfarabi College of Medicine, Saudi Arabia

10.1136/jgc-2020-IGCS.232 